\title{
Percepção de profissionais de saúde de CAPS I quanto a demandas relativas ao consumo de crack
}

\author{
Perception of health professionals from Psychosocial Care Centers \\ (CAPS I) regarding the demands related to the use of crack
}

Rogério Lessa Horta ${ }^{1}$

Georgius Cardoso Esswein ${ }^{2}$

Cristina Lessa Horta ${ }^{1}$

${ }^{1}$ Prontamente Clínica de Psiquiatria e Psicoterapia. Av. Desembargador Espiridião de Lima Medeiros 145, Três Figueiras. 91330-020 Porto Alegre RS. rogeriohorta@ prontamente.com.br ${ }^{2}$ Unisinos.

\begin{abstract}
This paper presents qualitative research analyzing two focus groups with twenty workers in Psychosocial Care Centers (CAPS I) in Porto Alegre, southern Brazil, in 2009. They were invited to discuss overwork and dissatisfaction with work in general and also recent demands of crack cocaine users on the local mental health networks. Workers in both groups reported a strong concern about the instability of employment contracts, leading to limited ability to plan their futures. In addition, the accumulation of tasks to replace absenteeism in teams leads to a limited interest in long-term action planning and the option for less complex tasks. Court orders, the interference of public officials and requests of hospitalization, are considered unfavorable routine conditions in the perception of health workers, as well as uncooperative and quarrelsome family members and unmotivated users for resuming treatment. The teams seem to be the center of a conflict between the impossibility of avoiding the emergence of demands of crack users who apply to local health networks and the limits established by management, increasing dissatisfaction and perceived overwork.
\end{abstract}

Key words Qualitative research, Job satisfaction, Psychosocial Care Centers, Mental health professionals
Resumo Pesquisa qualitativa que analisa dois grupos focais realizados em 2009 com vinte trabalhadores de dois CAPS I da região de Porto Alegre, para verificar a sua percepção diante do aumento de demandas que a expansão do crack tende a gerar sobre as redes locais de saúde mental. Propôs-se que debatessem o que lhes parecia fonte de sobrecarga e insatisfação em geral e, especificamente, em função das demandas de usuários de crack. Os resultados mostram forte preocupação com a instabilidade dos contratos de trabalho, levando à falta de projeção no futuro. Além disso, o acúmulo de tarefas para suprir carências das equipes, justificando-se o limitado interesse em planejar ações de longo prazo e a opção por tarefas menos complexas. Consideraram-se problemas na rotina de trabalho as ordens judiciais e a interferência de agentes públicos, pedido expresso de hospitalização, familiares sem postura cooperativa, querelantes e usuários desmotivados para o tratamento ou reingressando no serviço. As equipes parecem estar no centro um conflito entre a impossibilidade de se evitar o surgimento de demandas da população usuária das redes locais de saúde, o que se evidencia no contexto do uso de crack, e os limites da gestão, ampliando o desgaste e a sobrecarga percebidos.

Palavras-chave Pesquisa qualitativa, Satisfação no trabalho, Centros de Atenção Psicossocial, Profissionais de saúde mental 


\section{Introdução}

O mercado do crack cresceu significativamente no Brasil, em especial na última década ${ }^{1}$. O crack suscita preocupação por dúvidas sobre a efetividade dos planos de tratamento e pela dificuldade de adequação da oferta de serviços à expansão da demanda, além de repercussões clínicas significativas entre seus usuários, como episódios psicóticos, déficit cognitivo, alterações do humor e do comportamento e pneumopatias, entre ou$\operatorname{tras}^{2-4}$. A atenção necessária à população usuária de crack, que se multiplica em curto espaço de tempo, demanda flexibilidade e capacidade de adaptação dos serviços de saúde, além de maiores investimentos na área ${ }^{5,6}$.

A Reforma Psiquiátrica Brasileira propôs os Centros de Atenção Psicossocial (CAPS) como referência das redes locais de saúde no sistema público brasileiro (SUS) e como equipamento extra-hospitalar capaz de oferecer atenção que integre, de modo efetivo, recursos clínicos e a dimensão social dos agravos em saúde mental ${ }^{7-9}$. Têm sido apontados alguns nós neste modelo, como a necessidade de mais investimentos e do aumento do seu número no país, além da busca por ampliação da qualidade do trabalho produzido lá, com capacitação dos profissionais, expansão dos níveis de satisfação e redução do nível de sobrecarga dos trabalhadores em atividade nesses serviços ${ }^{10-12}$.

O crack pode atuar como elemento de desequilíbrio neste processo e no debate em torno dele. Os níveis de satisfação e sobrecarga das equipes, sua qualificação técnica, a escolha de procedimentos, a efetividade dos recursos terapêuticos disponíveis, o estágio motivacional do sujeito que chega para o tratamento, a condição clínica de cada paciente e a condição de sua rede familiar são decisivos para promover adesão e efetividade terapêutica ${ }^{12-14}$.

Este artigo examina as condições em que as equipes de dois CAPS I de municípios de pequeno porte da região metropolitana de Porto Alegre se percebiam, em 2009, em relação à expectativa de demanda e às demandas existentes decorrentes de problemas associados ao uso de crack naquele momento.

\section{Método}

Trata-se de uma pesquisa qualitativa, na qual foram realizados, em 2009, dois encontros de discussão focal de grupos, com cerca de duas horas de duração, cada um com 10 profissionais (20 ao todo) de nível médio e de nível superior dos CAPS I de municípios de pequeno porte da região metropolitana de Porto Alegre (RS), Igrejinha e Taquara. Cada município tem um único CAPS I, que cumpre o papel de principal referência local em atenção em saúde mental.

O grupo focal permite simultaneamente colher dados e promover a reflexão de cada equipe acerca do tema em estudo ${ }^{8,15,16}$. Nos encontros realizados não se abordava diretamente as dificuldades no atendimento da população usuária do crack, evitando-se, assim, respostas decorrentes de mecanismos defensivos ou do constrangimento de membros do grupo. A proposta era de que o grupo discutisse a percepção da sua condição e das condições de trabalho, a partir de um aquecimento baseado na apresentação sucinta das subescalas de dois instrumentos: SATIS-BR, que avalia satisfação com subescalas para qualidade do serviço, participação no serviço, condições de trabalho e relacionamento com os colegas e superiores e IMPACTO-B, que avalia sobrecarga, com subescalas para impacto do trabalho sobre a saúde física e mental, impacto do trabalho sobre o funcionamento profissional e impacto do trabalho sobre o estado emocional ${ }^{12,17}$.

À medida que os grupos se motivavam e passavam à discussão de sua condição, os participantes eram desafiados a relacionar tal condição com a situação específica de enfrentamento das demandas geradas pela população usuária de crack naquele período.

Quando se percebia o esgotamento da produção de novos registros ou de movimentos de repetição das manifestações, encaminhava-se o encerramento dos encontros.

Os encontros foram gravados, transcritos e analisados, sob uma perspectiva de base fenomenológica, com vistas à identificação de emergentes grupais que descrevessem as principais dificuldades percebidas pelas equipes e suas expectativas diante das potencialidades também verificadas ao assumirem a tarefa de acolher usuários de crack e oferecer ações de cuidado específicas para esta população, de modo continuado, em seus municípios.

Este estudo foi aprovado pelo Comitê de Ética em Pesquisa da Universidade do Vale do Rio dos Sinos - Unisinos e desenvolvido com apoio financeiro do Conselho Nacional de Desenvolvimento Científico e Tecnológico (CNPq). 
A análise de emergentes grupais permite formular pelo menos três categorias, que contemplam potencialidades do trabalho em CAPS I e as dificuldades inerentes ao processo de sua organização ou execução. A partir do debate sobre carga de trabalho e satisfação, foram mencionadas percepções e também expectativas das equipes, que diziam respeito principalmente a:

- Condições de trabalho;

- Interesse e motivação das equipes;

- Características dos usuários nos acolhimentos.

São ainda raros estudos sobre os modelos substitutivos de atenção em saúde mental. Apesar das transformações em curso nos países da América Latina e das evidentes possibilidades e dificuldades inerentes a estes processos, apenas $4,4 \%$ das pesquisas em saúde mental na região correspondem a estudos sobre os sistemas de saúde e políticas públicas de saúde mental ${ }^{18}$. Entre os estudos disponíveis, vários dão conta da realidade dos CAPS, evidenciando que, uma vez instalados os serviços, prevalece a preocupação com as condições de trabalho, a qualidade dos recursos terapêuticos e com a efetividade das práticas clínicas disponíveis ${ }^{8,12,19}$. Parece haver, portanto, um período de transição entre a abertura de frentes de trabalho e a qualificação do cuidado. Os CAPS I visitados neste estudo parecem viver esta fase crítica e estabelecem representações e expectativas muito parecidas com as que outras equipes também evidenciaram, o que reforça o nexo que se descortina neste estudo entre as categorias formuladas, como veremos a seguir:

\section{Condições de trabalho}

As equipes destacaram o valor de se levar alternativas de cuidado em saúde mental para os municípios de portes como os deste estudo, com acesso facilitado à população e seguimento dos cuidados na própria comunidade. As equipes reconhecem e valorizam os esforços da gestão pública no sentido de garantir, por exemplo, orçamento público para o seguimento de seu trabalho, mas indicam que os limites hoje estabelecidos impõem carências e insuficiências importantes:

... a gente sabe que é melhor ter o serviço aqui, é importante...

...o secretário sempre diz que a gente é prioridade, que não vai deixar de ter recurso...

...eu não duvido que eles fazem o melhor, mas falta muita coisa..
Segundo a literatura revisada, isso não ocorre apenas nos municípios sede desta pesquisa. Uma fase inicial de investimentos no comprometimento dos diferentes níveis de gestão, levando responsabilidades e competências no cuidado em saúde mental até os menores municípios, para que se tenha atendimento próximo do domicílio dos usuários se mostrou benéfica e ainda é necessária, embora permeada de insuficiências ${ }^{9,11,18,20}$.

Três temas foram mais relevantes na formação da categoria condições de trabalho: a insegurança manifesta pelos participantes quanto à sua inserção nas equipes, a precarização das condições de trabalho e a participação das cooperativas de trabalho neste processo, além do relacionamento com gestores e sua limitada participação em níveis de gestão.

Os profissionais destes CAPS I se percebiam ameaçados por contratos de curta duração, intermediados por cooperativas nas quais não tem uma participação societária real e efetiva e sem garantias de continuidade na prestação de serviço:

...dizem que é cooperativa, mas a gente nem sabe quem que manda lá...

...assim como tá aqui, já não tá...

...eu quis saber como era a tal cooperativa, mas ninguém sabia dizer...

Além disso, os grupos informaram que há diversas modalidades de contratação em vigor de modo simultâneo no mesmo grupo de trabalho, com padrões salariais distintos, mas todos mencionados como insuficientes ou insatisfatórios:

...tem de todo o tipo: concursado, contrato, cooperativa...

...a gente ganha pouco, não é grande coisa...

Os grupos indicaram saber que os gestores tendem a manter pessoas em cada equipe e a manter os projetos em andamento, mas disseram não ter nenhuma convicção quanto à sua permanência, em particular, em seus postos de trabalho, enriquecendo o argumento com exemplos próprios de passagens por diversos outros serviços e pela constatação de que poucos no grupo poderiam informar estar ou ter estado por dois anos no mesmo posto ou na mesma função: ...eu mesma já trabalhei no posto lá do (bairro) e agora to aqui...

...tem umas pessoas que tão aqui desde o começo, mas eu já tive, saí e agora to de volta...

...ninguém sabe onde vai tá daqui um tempo...

...é por um ano, quando renova o contrato, se renova, aí é que a gente sabe prá onde vai, é prá onde tá precisando naquela hora...

As cooperativas de trabalho, através das quais alguns profissionais são contratados, foram men- 
cionadas como formas de contratação precárias e de condições desfavoráveis. A percepção dos grupos a respeito desta solução administrativa pareceu negativa. Os grupos sugeriram, inclusive, que não seriam realmente instituições de caráter cooperativo, principalmente por não reconhecerem ali a possibilidade de participação deliberativa ou diretiva. A insegurança em relação às cooperativas estava associada à falta de informações, que apareceu também de outras formas. Nos dois grupos foi indicado que tinham dificuldade de saber em detalhes o que era planejado ou proposto numa perspectiva de médio ou longo prazo em termos de gestão dos serviços locais de saúde. Referiram também não ter muitas informações sobre os contratos vigentes entre o poder público e as empresas que os contratavam, não conhecer detalhes dos acordos, como vigência, valores repassados e nem quanto à regularidade dos recolhimentos e obrigações trabalhistas:

...eu nunca fui chamada..

„,nunca soube de assembleia, nem sei quem dirige isso.. disso...

...não sei de nada, a gente não fica sabendo nada

Além disso, o processo de contratação vigente gera também rotatividade no grupo de servidores. Os participantes referiram ser fácil qualquer pessoa, de qualquer das equipes, aceitar convite para inserção profissional em outro serviço ou até em municípios vizinhos. $\mathrm{Na}$ fala dos participantes, os salários são baixos o suficiente para logo se interessarem por outros contratos:

...é o que a gente tem, mas se aparecer outra oportunidade, eu vou, porque aqui pagam pouco...

...se pagar melhor, fazer o quê? Aqui não tem nenhuma garantia...

A forma de contratação, as prioridades em termos de planejamento e a destinação de recursos são temas que permeiam esta discussão e passam pelos níveis de gestão pública, em relação aos quais apareceram neste estudo referências à pouca autonomia do trabalhador, participação apenas limitada e eventual, além de diálogo limitado com as pessoas envolvidas em cargos diretivos:

...na verdade a gente não decide..

...o planejamento muda de acordo com o que vem da secretaria..

... a gente tem que fazer o que dá..

.... g gente não sabe muito bem...

...o secretário até vem aqui, conversa com a gente, mas quase sempre é prá comunicar coisas..

Não se pode dizer que os trabalhadores reunidos neste estudo se percebem atuando como cogestores das redes locais de saúde. Como em outras regiões do país, as equipes percebem os processos de gestão como pouco democráticos e nada participativos ${ }^{13}$. Cogestão não impede que se estabeleçam condições precárias de contratação, mas um processo de gestão mais participativo sequer é viável com relações trabalhistas pouco duradouras e sem nenhuma garantia de permanência no vínculo com o serviço de saúde.

A precarização das condições de trabalho já é condição reconhecida como parte do processo de expansão da atenção em saúde mental ${ }^{13}$. Envolve salário insatisfatório e contratos temporários, onde há menor valorização da força dos trabalhadores, bem como maior número de horas de trabalho semanal. Aparecem também insegurança, instabilidade e vínculos fragilizados, com direitos trabalhistas limitados ao básico da legislação vigente e o descomprometimento da gestão quanto à estabilidade dos contratos, sendo pouco frequentes as contratações via concurso público ${ }^{13,19}$. Há inúmeras condições potencialmente determinantes da precarização, entre elas, vicissitudes do processo político e as opções estratégicas assumidas, que envolvem eleição de prioridades e distribuição dos recursos disponíveis, além dos limites legais para o investimento em saúde ${ }^{20}$. Há também repercussões evidentes sobre o exercício profissional e o resultado das práticas de cuidado.

\section{Interesse e motivação das equipes}

As dificuldades relacionadas às condições de trabalho foram referidas como induzindo ou, pelo menos, justificando, pouco interesse em planejar ações de longo prazo e pouco comprometimento, disponibilidade ou envolvimento em ações mais complexas ou de resultados menos imediatos. Os profissionais se perceberam também menos dispostos ao envolvimento no planejamento do serviço do que já haviam experimentado em outros momentos ou em outros serviços, indicando contar com habilidades técnicas e experiência pessoal prévias com potencial para maior aproveitamento do que se percebe nas condições atuais. Habilidades e experiências que se tornam indisponíveis, seguidas de referências à percepção de que os serviços operam com sobrecarga, com limitação do planejamento ao curto prazo, se traduz externamente como evidência de menor capacidade técnica das equipes e confunde a avaliação das reais necessidades de capacitação e qualificação profissional.

Os grupos referiram ter formação específica para o trabalho ou experiência em práticas de 
cuidado continuado e inserção em redes de cuidado nas comunidades, pelo menos para a maioria dos membros das equipes:

...na verdade a gente sabe o que tem que fazer...

...muitos de nós já trabalharam aqui ou em outros municípios...

.... a gente aprendeu muito com os pacientes graves, não os usuários de drogas, as pessoas com esquizofrenia, por exemplo...

... a gente sabe, por exemplo, que visita domiciliar pode ajudar a trazer a pessoa, mas cadê? Quem vai fazer?

...fez diferença, quanta gente custou a se firmar aqui, quantas vezes a gente ia na casa, procurava, falava com as pessoas que moram ali...

As equipes reconhecem a possibilidade de realizar mais tarefas e algumas de maior complexidade, como busca de pacientes que interromperam ou abandonaram o atendimento nos CAPS I, visitas domiciliares, entre outras. Ressaltaram que as tarefas são conhecidas dos grupos porque já foram ou ainda são realizadas, mas sem a regularidade, sem a devida inserção no planejamento do serviço e sem o empenho que julgam mais adequados. Estas tarefas implicam ambições de longo prazo das equipes, como o investimento em vínculos fortes com as comunidades.

Os participantes se disseram sobrecarregados pelas tarefas que assumem rotineiramente e incapazes de acreditar em sua própria permanência naqueles serviços, ou mesmo nos municípios, por longo prazo. Isso estimula planejamento de ações de prazo mais curto. Não apenas o acúmulo de tarefas na tentativa de manter o maior número possível de atividades em funcionamento, a despeito do número de integrantes dos grupos, apareceu como decisiva para um planejamento de curto prazo, mas a reduzida perspectiva em relação ao futuro, discutida acima, também contribui. As equipes reconheciam, naquele momento, mecanismos defensivos priorizando eleição de tarefas com objetivos menores e resposta mais imediata:

.... g gente se vira, faz o que dá...

...não dá prá pensar muito adiante...

...não tem muito espírito prá planejar e pensar se a realidade tá aí, é a agenda cheia...

...eu to na reunião e to preenchendo documentos, fazendo anotação em prontuário...

Ao mesmo tempo, descreviam um movimento no sentido de preservar o funcionamento dos CAPS I e tentar dar conta da demanda identifica$\mathrm{da}$, suprindo eles mesmos os afastamentos, as ausências e a vacância de alguns cargos. Os grupos atribuíram este fato ao reduzido número de profissionais nas equipes e a incertezas quanto à substituição de quem sai:

$$
\text { ...faltagente... }
$$

...tem que esperar, mas demora, tá fazendo falta...

...agora mesmo, tá saindo a (nome) e não veio ninguém ainda...

...sai uma pessoa, a (nome), por exemplo, eu assumo a agenda dela até vir outra, mas vai demorando, dai se precisar fazer qualquer outra coisa, como é que eu faço?

...demora prá vir outra pessoa, a gente não sabe como fica...

Os desdobramentos de sua condição de inserção nos serviços podem levar a um posicionamento das equipes como incapazes de acolher as demandas da população de usuários de crack ou qualquer outra, algumas vezes por dificuldade técnica, como dúvidas sobre como proceder, mas eventualmente por indisponibilidade, muitas vezes expressa nas agendas. Isso gera alguma confusão porque as limitações impõem simplificação dos processos de trabalho, o que dificulta a identificação das deficiências técnicas e carências em termos de qualificação profissional das equipes. Segundo os participantes, quando se chega ao registro de que não há disponibilidade para atendimentos mais frequentes, as equipes já estão em condição muito crítica, já deram cobertura a inúmeras deficiências e já tentaram reorganizar os processos de trabalho:

... a gente fica sem saber o que fazer...

... a gente tenta, abre mais uma vaga no grupo, um horário extra, mas tem hora que não tem jeito...

...e se não tem como encaixar, o que que a gente faz?

...se eu pego os pacientes dela (profissional que saiu) prá não pararem o tratamento e não vem ninguém, como eu vou agendar mais gente?

Isso fica ainda mais evidente quando se percebe que tarefas como o atendimento a famílias, visitas domiciliares, busca de pacientes que interrompem o tratamento e atividades de planejamento ou reuniões de equipe não aparecem na grade de atividades dos profissionais, ainda que algumas delas sejam realizadas até com regularidade (as reuniões, por exemplo):

...tem o grupo de familiares, naquele horário...

...terapia de família ou atendimento das famílias, assim, cada uma, não tem mesmo, só no acolhimento, ou algum atendimento eventual, quando precisa...

...se tem que fazer uma visita, aí eu tenho que ir, né, no fim do dia... 
...por enquanto não tem quem faça (atendimento de famílias ou visita domiciliar), a gente tá vendo isso...

...reunião tem sempre, mas não dá prá deixar de fazer as outras coisas...

...é assim, a gente tá aqui, mas as pessoas tão chegando, tão ali, batem na porta...

Diante da expansão do mercado de crack, mesmo reconhecida a especificidade das demandas trazidas aos serviços por usuários e seus familiares, os participantes relatam não ter havido esforços específicos de revisão do planejamento, nem internamente, nem com os níveis de gestão mais acessíveis a eles:

...chega no acolhimento e a gente avalia...

...tem os grupos, as oficinas, a gente vê se tem condições de participar...

...não tem um programa específico, nem prá isso, nem prá risco de suicídio, a gente não consegue...

Não reagir de modo particular a demandas específicas aparece como uma decorrência da menor disponibilidade, que parece reduzir também os movimentos de aproximação com outros serviços da rede e a dinâmica de trabalho articulado numa rede efetiva. Isto reaparece nos limites que os profissionais referem como sobrecarga, pois à medida que as redes locais são mais frágeis os CAPS I ficam mais isolados:

...no posto de saúde a gente nunca sabe quemé o médico...

...tem uns colega que recebem bem e participam, mas outros nem ligam...

.... a gente faz o matriciamento e tem uns serviços onde é bem legal, mas não dá prá contar com todos...

.... a gente encaminha os pacientes, mas é muito difícil, o pessoal recebe mal...

...tem uns que só dão o papel prá vir prá cá, se a gente tenta falar prá discutir o caso, nem dá...

Os procedimentos e as técnicas a serem aplicados são definidos de acordo com capacidades anteriormente apropriadas pelos profissionais e isso também ocorre a outras equipes ${ }^{20}$. Pode-se dizer que não se estabelece um conjunto específico de reações para cada uma das grandes questões nos serviços. Se não são previstas ações ou reações específicas para situações mais prevalentes ou muito específicas, não há como serem estabelecidas medidas de avaliação de efetividade para cada uma ou para o conjunto das ações dos serviços. Sem avaliação, permanece nebulosa a definição de deficiências e a necessidade de qualificação.

Os participantes dos grupos se dizem capazes de realizar tarefas essenciais para o tipo de cuidado que as demandas geradas por usuários de drogas como o crack mobilizam, mas se percebem indisponíveis para tais atividades. Isso se aproxima da inexistência de limite definido para o número de usuários para cada profissional de referência, descrita por Miranda e Campos ${ }^{8}$. Os autores também relataram dificuldades para o desenvolvimento de um trabalho efetivamente integrado por diferentes abordagens, numa perspectiva multiprofissional, que deveria ter um profissional de referência como articulador do projeto terapêutico individual mais elaborado, complexo e compreendendo técnicas ou abordagens não necessariamente empregadas por este profissional. Isso cria uma espécie de simplificação da tarefa, não isenta de rebaixamento em termos de objetivos a longo prazo. Isto é semelhante ao que os participantes dos grupos deste estudo mencionaram como uma opção por tarefas menos complexas e menos envolventes em termos de planejamento de longo prazo. Reaparece aqui forte pressão na interface das equipes com os níveis de gestão, que é apenas parcialmente atenuada por esforços diversos, gerando o que outros profissionais em outras equipes de saúde também identificaram como sobrecarga ${ }^{20}$, especialmente vinculada ao acúmulo de tarefas.

Não foi referido por estes grupos de profissionais de CAPS I, mas o contexto referido aqui também pode levar ao que já aparece como uma tendência verificada em diferentes regiões do país: a permanência indefinida de usuários nos CAPS, instituindo um novo processo no estilo manicomial ou uma nova cronicidade para a doença mental, traduzida agora como a impossibilidade de usuários do serviço deixarem os CAPS ${ }^{9}$. Isso também define limites que garantem condições de sobrevida das equipes.

\section{Características desfavoráveis nos acolhimentos}

A cronificação da permanência em CAPS, se entendida como opção de redução de demandas sobre as equipes, assemelha-se ao descrito pelos participantes dos grupos focais deste estudo como características desfavoráveis dos usuários e de seus acompanhantes. $\mathrm{O}$ rechaço também pode indicar esforços espontâneos de adequação das demandas às disponibilidades das equipes. Algumas pessoas em algumas condições se tornam mais elegíveis para o atendimento que outras.

As equipes aparecem, assim, como o ponto de encontro de forças antagônicas: o rebaixamento das demandas esperado em função da 
precarização das condições de trabalho e da limitação de disponibilidades e a ampliação das demandas pelo surgimento de novos agravos ou aumento do número de casos de algum já existente, fenômenos típicos das dinâmicas envolvendo o binômio saúde-doença em qualquer comunidade. A emergência de novos casos em função do consumo de crack não é o primeiro e não será o último exemplo de explosão de demanda sobre as redes locais de saúde.

Ainda que o contexto das equipes não parecesse o mais favorável ao acolhimento de novas demandas, os grupos informaram já estar lidando com pessoas que buscavam atendimento por situações relacionadas ao consumo de crack. As equipes conseguiram indicar algumas condições dos acolhimentos que mais se associavam a insatisfação ou sobrecarga em suas atividades nos CAPS I. Referiam expectativas de que algumas características das demandas que se apresentavam pudessem ser evitadas, entre elas: os pedidos expressos de hospitalização, a relação com os familiares ou acompanhantes, pessoas que lhes causam impressões iniciais desagradáveis e o reingresso dos usuários de crack ou outras substâncias:

...tem gente que vem aqui e só quer o papel prá ir pro hospital...

...não querem esperar, perguntam se vai demorar...

...e se a gente diz que não precisa ir pro hospital?

...tem uns que nem ouvem a gente...

...vem uns de um jeito que não dá nem prá ficar na sala...

...e os conhecidos, então! Tem uns que já passaram umas quantas vezes por aqui, não ficam...

...tem uns que somem, mas a gente já sabe, daqui a pouco aparecem pedindo atendimento...

Os CAPS destes municípios têm papel regulador e suas equipes deliberam sobre a ocupação dos leitos de referência para a área da saúde mental. A exigência de leitos hospitalares que não considera a indicação do profissional que acolhe o paciente no CAPS foi descrita como eventual e tendo sua forma mais perturbadora nas ordens judiciais. Os pedidos oriundos ou encaminhados por gestores ou pessoas ligadas a grupos políticos influentes sobre a gestão pública também foram mencionados como perturbadores. Em ambos os casos, a reivindicação das equipes é para que se faça ouvir o grupo ou o profissional da equipe que faz o acolhimento do usuário. Apareceu a sugestão de que um acordo ou convenção promovesse uma uniformidade no sentido de se requerer ou ordenar a avaliação dos pacientes, não o procedimento a ser executado, mas apa- rentemente ninguém das equipes está cuidando do estabelecimento deste tipo de acordo:

...eles deviam pedir a nossa avaliação, não o leito...

...já vem o oficial de justiça com o paciente, não quer esperar e a ordem é de hospitalizar, daí a gente tem que parar tudo e sair atrás de vaga...

...e quando a gente já avaliou o caso e aí vem do juiz com ordem de baixar...

O oficial de Justiça é, portanto, o pior acompanhante possível na percepção dos profissionais, quando alguém chega para atendimento. A vinda de pessoas desacompanhadas não é oficialmente estimulada pelos CAPS I, mas os acompanhantes imaginados pelos participantes do estudo eram considerados potencialmente piores do que o paciente vir só. As equipes indicaram considerar importante que os familiares compareçam, mas costumam ter dificuldades no relacionamento com eles, não se sentem adequadamente preparados para lidar com familiares e não havia nas equipes, à época dos encontros, profissionais treinados para trabalho terapêutico com famílias. Não foram descritos também muitos espaços ou atividades planejadas especificamente para as famílias. Como não é raro que os mesmos façam reclamações, críticas ou tragam demandas diversas, como expectativas de resultados mais expressivos, com menor envolvimento ou maior brevidade, acabam sendo percebidos como sobrecarga ou ameaça de sobrecarga.

Os pacientes e seus acompanhantes causam impressões iniciais de diferentes impactos nos profissionais, variando de agradável e que denota interesse pelo serviço e pelo tratamento até rechaço ou repulsa, mais frequentemente associados a condições indicativas de pouca higiene corporal e de vestuário, postura hostil ou arrogante ou evidente desinteresse pelo serviço oferecido, o que os participantes perceberam como mais comum entre usuários trazidos contra a vontade ou naquela situação de procura direta e exclusiva por leito hospitalar.

O desinteresse pelo tratamento também é associado ao fato dos pacientes interromperem seus atendimentos e, mais adiante, retornarem. As equipes percebem este comportamento como hostil e caracterizando pouco aproveitamento do esforço despendido para gerenciar cada caso. Os profissionais disseram saber, racionalmente, que usuários de drogas tendem a interromper os tratamentos e reingressar, mas a expectativa é de que não ocorra. As reentradas dos usuários no mesmo serviço foram mencionadas como indicando menor grau de motivação para mudança 
de comportamento em relação à substância ou maior gravidade das repercussões decorrentes de seu uso. Os reingressos pareciam, segundo os grupos, refletir as recaídas (retorno ao consumo) dos pacientes usuários de drogas, gerando expectativas de baixa adesão ao tratamento, menor possibilidade de resultados satisfatórios, mais reingressos ainda, novos acolhimentos e uma tendência a ficar reiniciando o trabalho, portanto, mais desgaste e insatisfação das equipes. Esta condição tornaria o usuário menos merecedor dos investimentos da equipe:

...tem uns que não adianta...

... a gente já sabe, fica um pouco e some...

...vem quando tá pior, pede ajuda e depois logo some...

As equipes que participaram deste estudo referiram não ter conhecimento de acordo com o Poder Judiciário local a respeito das ordens judiciais de tratamento ou de hospitalização, apesar de descreverem algumas iniciativas próprias neste sentido, com encontros ou reuniões em algum momento. Indicaram também não ter nenhum tipo de mobilização regular em torno das questões de gestão ou dos contratos de trabalho, apesar de terem algum grau de proximidade com os gestores, nos dois municípios, mas apenas através dos canais institucionais formais, não como ação corporativa. As equipes vinham usufruindo de supervisões clinico institucionais promovidas pelo Ministério da Saúde, mas não demonstraram nenhuma experiência ou aprofundamento em termos de planejamento detalhado de capacidades instaladas, insuficiências e uma apropriação das consequências imediatas, por exemplo, da retirada de um dos membros de cada equipe. Não tinham, por exemplo, um mapa de necessidades pronto no momento em que os grupos focais ocorreram.

Ainda em fase de implantação, como política nacional, e precisando superar as contradições e as falhas do processo, as condições referidas pelos trabalhadores que participaram dos grupos deste estudo também aparecem como dificuldades em estudos com trabalhadores de CAPS em outras regiões do Brasil ${ }^{8,13,19-21}$.

Os CAPS e as redes locais de saúde se deparam com as demandas oriundas da expansão do mercado de crack numa condição ainda muito sensível. Quanto mais limitada a capacidade de expansão de um serviço e quanto menor a disponibilidade das equipes, menor a flexibilidade para lidar com os desdobramentos de mudanças $^{8,9,11,13,20}$, como as que necessariamente decorrem de alterações do perfil epidemiológico dos agravos. Isso pode contribuir para que as equipes se tornem mais exigentes e menos tolerantes às características e às condições de chegada ao acolhimento de seus usuários. A população que gera as demandas em saúde não encontra no cenário de precarização dos contratos de trabalho um mecanismo efetivo de regulação ou prevenção de riscos e agravos em saúde. A indisponibilidade eventual de uma equipe não limita a ocorrência de agravos à saúde da população assistida pelo serviço.

A comunidade é incapaz de evitar a geração de novas demandas ou maior carga de trabalho, maior envolvimento e maior desgaste para as equipes.

Outro conflito de difícil solução parece ser a reduzida tolerância das equipes às demandas que se impõem pelo envolvimento do Poder Judiciário, bem como ao reingresso de usuários. Usuários de substâncias psicoativas tendem a retornar aos serviços, interromper e retomar seus tratamentos até que atinjam graus de motivação suficientes para promover uma efetiva mudança de comportamento em relação ao consumo da droga ${ }^{22}$. Atingir níveis de motivação considerados prontidão para a mudança exige o emprego de um vasto elenco de técnicas, gerando condições para o processo terapêutico e ampliação ainda maior das possibilidades de intervenção. A aplicação de tais recursos depende de efetiva capacitação das equipes e, ainda assim, tende-se a trabalhar com lapsos, recaídas e reingres$\operatorname{sos}^{3,6,22,23}$. Ampliar a qualidade técnica das equipes pressupõe investimento, portanto, ampliação de demandas sobre gestores.

\section{Considerações Finais}

A partir do estudo das condições e dificuldades dos trabalhadores destes CAPS I, é possível a confirmação de que a sobrecarga e o sentimento de insatisfação, relatado pelos trabalhadores, comprometem a qualidade de trabalho impondo limites, tornando a autonomia precária e bastante reduzida a capacidade de desenvolver tarefas mais complexas, que ampliem a qualidade do serviço.

A precarização do trabalho propõe uma tendência ao rebaixamento de demandas que a população que busca atendimento não é capaz de seguir.

A promoção de ações de cuidado com os cuidadores deve indicar a ruptura desta tendência, pela promoção de um planejamento mais amplo 
das atividades das equipes e da avaliação detalhada de suas condições de trabalho, reduzindo o conflito potencialmente emergente diante da renovação e complexificação inevitáveis das demandas em saúde.

\section{Colaboradores}

\section{Referências}

RL Horta, GC Esswein e CL Horta participaram igualmente de todas as etapas de elaboração do artigo.

1. Carlini EA, Galduróz JC, Noto AR, Carlini CM, Oliveira LG, Nappo SA, Moura YG, Sanchez ZVDM. II Levantamento Domiciliar sobre o Uso de Drogas Psicotrópicas no Brasil: estudo envolvendo as 108 maiores cidades do país, 2005. São Paulo: CEBRID, UNIFESP; 2007.

2. Kessler F, Pechansky F. Uma visão psiquiátrica sobre o fenômeno do crack na atualidade. Rev. psiquiatr. Rio Gd. Sul 2008; 30(2):96-98.

3. Oliveira G, Nappo SA. Caracterização da cultura de crack na cidade de São Paulo: padrão de uso controlado. Rev Saude Publica 2008; 42(4):664-671.

4. Sanchez ZVDM, Nappo SA. Seqüência de drogas consumidas por usuários de crack e fatores interferentes. Rev Saude Publica 2002; 36(4):420-430.

5. Araujo RB, Oliveira MS, Pedroso RS, Miguel AC, Castro MGT. Craving e dependência química: conceito, avaliação e tratamento. J. Bras. Psiquiatr. 2008; 57(1):57-63.

6. Cruz MS, Silva F, Ferreira J. A formação de profissionais para a assistência de usuários de drogas e a constituição de um novo habitus de cuidado. J. Bras. Psiquiatr. 2005; 54(2):120-126.

7. Borges CF, Baptista TWF. O modelo assistencial em saúde mental no Brasil: a trajetória da construção política de 1990 a 2004. Cad Saude Publica 2008; 24(2):456-468.

8. Miranda L, Campos RTO. Análise do trabalho de referência em Centros de Atenção Psicossocial. Rev Saude Publica 2008; 42(5):907-913.

9. Pande MNR, Amarante PDC. Desafios para os Centros de Atenção Psicossocial como serviços substitutivos: a nova cronicidade em questão. Cien Saude Colet 2011; 16(4):2067-2076.

10. Kerr-Corrêa F. Dependência de Substâncias psicoativas: opinião. Psiquiatria Hoje 2008; 1:30-31.

11. Mari JJ. Uma chamada de ação global para o avanço da saúde mental. Psiquiatria Hoje 2007; 6:7.

12. Rebouças D, Abelha L, Legay LF, Lovisi GM. O trabalho em saúde mental: um estudo de satisfação e impacto. Cad Saude Publica 2008; 24(3):624-632.

13. Guimarães JMX, Jorge MSB, Assis MMA. (In)satisfação com o trabalho em saúde mental: um estudo em Centros de Atenção Psicossocial. Cien Saude Colet 2011; 16(4):2145-2154.

14. Williams AV, Meyer E, Pechansky F. Desenvolvimento de um jogo terapêutico para prevenção da recaída e motivação para mudança em jovens usuários de drogas. Psic.: Teor. e Pesq. 2007; 23(4):407-413. 
15. Moraes $\mathrm{M}$. O modelo de atenção integral à saúde para tratamento de problemas decorrentes do uso de álcool e outras drogas: percepções de usuários, acompanhantes e profissionais. Cien Saude Colet 2008; 13(1):121-133.

16. Nunes M, Torrenté M, Ottoni V, Moraes Neto V, Santana M. A dinâmica do cuidado em saúde mental: signos, significados e práticas de profissionais em um Centro de Assistência Psicossocial em Salvador, Bahia, Brasil. Cad Saude Publica 2008; 24(1):188-196.

17. Bandeira M, Ishara S, Zuardi AW. Satisfação e sobrecarga de profissionais de saúde mental: validade de construto das escalas SATIS-BR e IMPACTO-BR. J. Bras. Psiquiatr. 2007; 56(4):280-286.

18. Razzouk D, Sharan P, Gallo C, Gureje O, Lamberte EE, Mari JJ, Mazzotti G, Patel V, Swartz L, Olifson S, Levav I, de Francisco A, Saxena S; WHO-Global Forum for Health Research Mental Health Research Mapping Project Group. Scarcity and inequity of mental health research resources in low-and-middle income countries: A global survey. Health Policy 2010; 94(3):211-220.

19. Rézio LA, Oliveira AGB. Equipes e condições de trabalho nos centros de atenção psicossocial em Mato Grosso. Esc Anna Nery 2010; 14(2):346-354.

20. Nascimento AF, Galvanese ATC. Avaliação da estrutura dos centros de atenção psicossocial do município de São Paulo, SP. Rev Saude Publica 2009; 43(Supl. 1):8-15.

21. Hirdes A. A reforma psiquiátrica no Brasil: uma (re)visão. Cien Saude Colet 2009; 14(1):297-305.

22. Oliveira MS, Laranjeira R, Araújo RB, Camilo RL, Schneider DD. Estudo dos Estágios Motivacionais em Sujeitos Adultos Dependentes do Álcool. Psico. Reflex. Crit. 2003; 16(2):265-270.

23. Garrett J, Stanton MD, Landau J, Baciewicz G, Brinkman-Sull D, Shea R. The "Concerned Other" Call: Using Family links and networks to overcome resistance to addiction treatment. Subst Use Misuse 1999; 34(3):363-382.

Artigo apresentado em 24/10/2011

Aprovado em 25/01/2012

Versão final apresentada em 08/02/2012 